\title{
ASYMMETRICAL DELAYED DETONATION FROM A 3D HYDROSIMULATION OF A WHITE DWARF EXPLOSION
}

\author{
E. BRAVO, AND D. GARCíA-SENZ
}

Dpt. Física i Enginyeria Nuclear, UPC, Barcelona, Spain, and Institut d'Estudis Espacials de Catalunya, Barcelona, Spain

The White Dwarf Delayed Detonation model for SNIa has been investigated using an SPH code. The relevant features and results are:

- The code is able to handle deflagrations as well as detonations.

- The code incorporates a nuclear network of 9 nuclei, from ${ }^{1} \mathrm{H}$ to ${ }^{56} \mathrm{Ni}$. The final nucleosynthesis comes from a post-processing calculation.

- During the deflagration phase the combustion front is increasingly deformed, but the effective velocity of the flame is too low to unbind the whole white dwarf. When the density at the flame reaches $\sim 5 \times 10^{7} \mathrm{~g} / \mathrm{cm}^{3}$ the fractal dimension of the flame surface at selected points is greater than 2.5. These points are then artificially incinerated, starting the detonation phase.

- The shock waves launched by the incineration of the high fractal dimension regions are able to induce detonation waves in the fresh fuel only where the environmental conditions are propitious (geometry, density, etc).

- The final composition is rich in C-O $\left(0.76 M_{\odot}\right)$, whereas the ejected mass of ${ }^{56} \mathrm{Ni}\left(0.26 M_{\odot}\right)$ is slightly too low for a SNIa. The detailed composition is underabundant in C-O, and has nearly Solar System proportions of ${ }^{32} \mathrm{~S},{ }^{36} \mathrm{Ar},{ }^{40} \mathrm{Ca},{ }^{48} \mathrm{Ti},{ }^{52,53} \mathrm{Cr},{ }^{55} \mathrm{Mn},{ }^{56,57} \mathrm{Fe},{ }^{60,61,62} \mathrm{Ni}$, and ${ }^{64} \mathrm{Zn}$, while ${ }^{54} \mathrm{Fe}$ and ${ }^{58} \mathrm{Ni}$ are a factor $4-5$ in excess.

- As a result of the asymmetrical propagation of the detonations, the typical distribution of abundances in the velocity space obtained in 1D models is not reproduced here. Thus, $\mathrm{Ni}, \mathrm{Si}$, and $\mathrm{S}$ are more abundant than $\mathrm{C}$ and $\mathrm{O}$ in the high velocity material, from $\sim 13,000$ to $27,000 \mathrm{~km} / \mathrm{s}$. Between 3,000 and $\sim 13,000 \mathrm{~km} / \mathrm{s} \mathrm{C}, \mathrm{O}$, and $\mathrm{Ni}$ are the dominant nuclei, while at lower velocities the composition is rich in $\mathrm{Fe}$.

As a conclusion, the feasibility of the computation of a Delayed Detonation in 3D has been shown. Our results show that the multidimensional properties of the combustion front must be carefully taken into account, which can not be done with a 1D code. Obviously, much work remains to be done. 